\title{
Improved PCA Method Based on RBF Neural Network for Multiple Response Parameters Optimization
}

\author{
Jianli Yu*, Xiaotian Pan and Hongqi Huang \\ Management Engineering Department, Zhengzhou University of Aeronautical, Zhengzhou, China \\ Corresponding Email: yj1837@163.com
}

\begin{abstract}
In the design of multiple response parameters optimization, weighted principal component analysis (weighted PCA) is used to build the relationship between the response variables and controllable factor model by linear regression. But in the complicated nonlinear production process, the fit of the linear regression model is not high that cannot satisfy the requirement of the parameter design model. This study proposed an improved weighted PCA based on RBF neural network prediction model. In this paper, RBF neural network was used to construct nonlinear prediction model of production process and to adjust the weighted PCA algorithm by adding the predict ability index of neural network model. In the design of multiple response parameters, this approach improve the effect of process parameters optimization. And applied this method to multiple response parameters optimization design of metallization polypropylene film capacitor thermal polymerization process, the results show that capacitance value and the loss tangent are all improved, and the effect of optimization parameters is achieve to satisfactory results.
\end{abstract}

\section{Introduction}

With the complexity of the production process and product quality requirements, it is need to consider multiple quality characteristics in the process of product optimization. The multiple quality parameters optimization method is aim to improve the products quality by process parameters optimization. So the multiple quality characteristics optimization design has a important role in the real process of quality continuous improvement.

The quality loss function and satisfaction function method have an extensive application in the multiple response optimization design, but it ignores the correlation between the response variables, which will affect the effect of optimization design [1]. So the problem of the correlation between the multiple quality characteristics caused more attention among the academics. Wang Jianjun, Ma Yizhong [2] used the partial least-squares regression model to solve the problem of the correlation between the multiple quality characteristics. Ali [3] proposed a new method of satisfaction function method and spherical rule model to solve the problem of correlation.

Liao [4] proposed weighted principal component analysis (PCA) which can combine the multiple response to the single response and eliminate the correlation between variables. In actual industrial production, PCA is applied to solve the multiple response parameters problem which have been achieved satisfactory results [5]. Zhang Yingdong [6] proposed an improved weighted principal component analysis(PCA) which can construct a mathematical model between the impact factor and multiple response variable. The traditional response surface method based on linear regression analysis to determine the the polynomial model of first-order or second-order. But the quality characteristics become more nonlinear and highly complicated in the modern advanced manufacturing progress, the traditional response surface based on linear model cannot meet the practical requirements [7]. However, artificial neural network can independently complete the nonlinear mapping from input space to the output space, it belong to nonlinear model which has the strong ability of prediction [8].

This paper proposed an improved PCA method based on radial basis function(RBF) neural network for multiple response parameters optimization. Firstly, construct a production process mapping relations between the factors and multiple response based on RBF neural network. Next, using PCA method eliminate the correlation between response variable through weighted multiple response variables into a single comprehensive response variable. Finally, it is priority to improve the response variable which have a strong prediction ability, and realize the overall effect of multiple response optimization. And applied this method for the metallized polypropylene film capacitor, which not only solving the parameters optimization problem of the polymerization temperature and polymerization time, but also realizing the whole optimization of the capacitor value and the loss tangent. 


\section{Improved PCA based on RBF neural network method}

\subsection{Radial basis function (RBF) neural network model}

Radial Basis Function (RBF) is an efficient feedforward neural network [9]. Its approximation ability and generalization ability and learning speed is all superior to the BP neural network [10]. RBF neural network can arbitrary approach to the linear and nonlinear function, so there is no local optimum problem. Its structure is shown in figure 1.

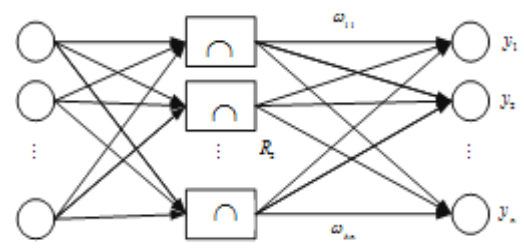

Fig. 1. The structure of RBF neural network.

In the complex nonlinear process, the fitting degree of the linear regression model is not high and the regression formula can not meet the forecast ability of the responses variable. Based on that, non-parametric model is established by using RBF neural network to forecast the process-response indicators, and the mean square error(MSE) as predictive ability evaluation index for RBF neural network model. The value of MSE is smaller, the RBF neural network model has a better prediction accuracy.

Assumes that the controllable factors variable is $\mathrm{x}_{1}, \mathrm{x}_{2}, \ldots \mathrm{x}_{\mathrm{i}}$, which as the input vector of RBF neural network model. And assume the total number of the response variable is $k$. The first $j$ response variable $y_{j}$ as output vector of $R B F$ neural network model. So the number of RBF model established is $\mathrm{k}$.

Using the MATLAB computer software to construct and train the RBF neural network model. The statements as following

$\mathrm{P}=\left[\mathrm{x}_{1} \mathrm{x}_{2} \cdots \mathrm{x}_{\mathrm{i}}\right]$ '; ' $\mathrm{x}_{1} 、 \mathrm{x}_{2} \cdots \mathrm{x}_{\mathrm{i}}$ 'is the controllable factors variable

$\mathrm{T}=\left[\mathrm{y}_{\mathrm{j}}\right]$ '; $\quad$ ' $\mathrm{y}_{\mathrm{j}}$ ' is the first $\mathrm{j}$ response variable

net $=$ newrb(p, t, goal, spread); $\mathrm{p}$ is the input vector, $\mathrm{t}$ is the output vector $\mathrm{y}=\operatorname{sim}($ net, $\mathrm{p})$;

$\mathrm{e}=\mathrm{T}-\mathrm{y}$; $\quad \mathrm{e}$ ' is the error vector between the expectations and forecast

$\mathrm{MSE}=\mathrm{mse}(\mathrm{e})$; $\quad \mathrm{mse}(\mathrm{e})$ ' is the mean square error

\subsection{Weighted principal component analysis}

Weighted principal component analysis method can combine multiple indicators into a new set of unrelated indicators, and according to the variance contribution get a weighted sum, which can transfer the multiple response to the single response.

Assumes that the number of the response is $\mathrm{p}$, and the response variable is $\mathrm{Y} 1, \mathrm{Y} 2, \ldots$, Yp. According to the principal component analysis, the principal component is $\mathrm{Z} 1, \mathrm{Z2}, \ldots, \mathrm{Zk}$. The formula of $\mathrm{Z1}, \mathrm{Z} 2, \ldots, \mathrm{Zk}$ is

$$
Z_{q}=e_{q 1} Y_{1}+e_{q 2} Y_{2}+\cdots+e_{q p} Y_{p}
$$

$\mathrm{Zq}$ is the principal component of first q. Yq is the response of the first q. "eq1,eq2,...,eqp" is the coefficient of the first q principal component. And the formula of the variance contribution rate is

$$
a_{q}=\frac{\lambda_{q}}{\sum_{q=1}^{k} \lambda_{q}}
$$

$\lambda_{q}$ is the eigenvalue of first $\mathrm{q}$.

Using MINITAB software to make principal component analysis that can get component load matrix and principal components variance contribution rate. According to the variance contribution rates can calculate a weighted sum of the principal component. And the formula of the multiple response performance index(MPI) is

$$
M P I=\sum_{q=1}^{k} \alpha_{q} Z_{q}
$$


By comparing the values of MPI can convert multiple performance characteristics optimization problem into a single response optimization problem. And if the goal or target of the response is larger for better, so the MPI is larger, the level of quality characteristics is higher. If the target of the response is smaller for better, so the MPI is smaller, the level of quality characteristics is higher.

\subsection{Improved weighted PCA method}

For RBF neural network prediction model, the corresponding mean square error (MSE) is an effective evaluation index for network prediction ability. So the predictive ability index of first $\mathrm{j}$. And the prediction ability of RBF neural network model is defined as

$$
R_{j}=\frac{\sum_{i=1}^{p} m s e_{i}-m s e_{j}}{\sum_{i=1}^{p} m s e_{i}}, r_{j}=\frac{R_{j}}{\sum_{i=1}^{p} R_{i}}, j=1,2, \cdots, p
$$

So the MSE is smaller, the predictive ability " $r$ " of RBF neural network model is higher.

Combining the RBF neural network model and weighted principal component analysis method, the formula of predict the response (1) is revised as follows

$$
Z_{q-\text { new }}=e_{q 1} r_{1} Y_{1}+e_{q 2} r_{2} Y_{2}+\cdots+e_{q p} r_{p} Y_{p}
$$

$\mathrm{eq}_{1}, \mathrm{eq}_{2}, \ldots, \mathrm{eq}$ is the coefficient of the first $\mathrm{q}$ principal component, $\mathrm{r}_{1}, \mathrm{r}_{2}, \cdots, \mathrm{r}_{\mathrm{p}}$ is prediction ability of RBF Neural network.

\section{The parameter optimization design of metallized polypropylene film capacitors}

The process of thermal polymerization is key to eliminating the air between the metallized film layers, which can improve the compactness of the capacitor and the stability of electrical performance. But if thermal polymerization is insufficient, which will drop down the capacity of capacitor and increase the loss tangent [11]. Therefore, it is necessary to optimization the two parameters of temperature and time in the process, which is expected to improve the capacitance value and loss tangent value.

In this paper, there is two responses in the optimization process of metallized polypropylene film capacitors. One of the response variables is the capacitance value(y1), the other response variable is loss tangent(y2). The target of capacitance value $(\mathrm{y} 1)$ is $45.45 \mu \mathrm{F}$, and the target of loss tangent $(\mathrm{y} 2)$ is smaller for better. So the absolute value of the difference between the capacitance value $(\mathrm{y} 1)$ and the target value $(45.45 \mu \mathrm{F})$ is converted to the smaller for better. And assume that the controllable factors variable of thermal polymerization temperature is $\mathrm{x} 1$, the polymerization time is $\mathrm{x} 2$. The process parameters and their level given in table 1.

Table 1. The process parameters and their level.

\begin{tabular}{|c|c|c|c|c|c|c|c|c|}
\hline No & parameters & Level 1 & Level 2 & Level 3 & Level 4 & Level 5 & Level 6 & Level 7 \\
\hline 1 & Temperature & 95 & 100 & 105 & 110 & 115 & 120 & 125 \\
\hline 2 & Time(h) & 4 & 6 & 8 & 10 & None & None & None \\
\hline
\end{tabular}

There are 28 groups of thermal polymerization experiments on metallized polypropylene film capacitor. In the level of $(1,1) 、(1,2) 、(1,3) 、(1,4) 、(2,1) 、(2,2) 、(2,3) 、(2,4) 、(3,1) 、(3,2) 、(3,3) 、(3,4)$ repeat the experiment 10 times respectively, other levels repeated the experiments 5 times respectively. The average of the two response variables in each experiment was calculated, so the experimental results are shown in table 2. Using the MINITAB to make the principal component analysis of response variables, and the eigenvalues and eigenvectors are shown in table 3.

Table 2. The experimental data and MPI.

\begin{tabular}{|c|c|c|c|c|c|c|c|c|c|}
\hline \multirow{2}{*}{$\begin{array}{c}\text { Experi } \\
\text {-ment } \\
\text { No. }\end{array}$} & \multicolumn{2}{|c|}{$\begin{array}{c}\text { Factor } \\
\text { variable }\end{array}$} & \multicolumn{2}{|c|}{$\begin{array}{c}\text { Response } \\
\text { mean }\end{array}$} & \multicolumn{2}{c|}{$\begin{array}{c}\text { Standardized } \\
\text { mean }\end{array}$} & $\begin{array}{c}M P I_{1} \\
\text { (first-order } \\
\text { regression) }\end{array}$ & $\begin{array}{c}M P I_{2} \\
\text { (second- } \\
\text { order } \\
\text { regression) }\end{array}$ & $\begin{array}{c}M P I_{3} \\
(\mathrm{RBF})\end{array}$ \\
\hline 1 & 1 & 1 & 0.2051 & 3.8 & 0.534 & 1.081 & 0.3751 & 0.2901 & 0.3702 \\
\hline 2 & 1 & 2 & 0.0331 & 3 & -0.703 & -1.390 & -0.4939 & -0.3817 & -0.4874 \\
\hline 3 & 1 & 3 & 0.0383 & 3.4 & -0.666 & -0.154 & -0.4674 & -0.3521 & -0.4607 \\
\hline 4 & 1 & 4 & 0.0101 & 3.6 & -0.869 & 0.463 & -0.6096 & -0.4540 & -0.6006 \\
\hline 5 & 2 & 1 & 0.1268 & 3.6 & -0.029 & 0.463 & -0.0204 & -0.0117 & -0.0199 \\
\hline 6 & 2 & 2 & 0.0336 & 3.1 & -0.700 & -1.081 & -0.4913 & -0.3773 & -0.4847 \\
\hline
\end{tabular}




\begin{tabular}{|c|c|c|c|c|c|c|c|c|c|}
\hline 7 & 2 & 3 & 0.0045 & 3.7 & -0.909 & 0.772 & -0.6378 & -0.4728 & -0.6283 \\
\hline 8 & 2 & 4 & 0.0063 & 3.3 & -0.896 & -0.463 & -0.6290 & -0.4758 & -0.6202 \\
\hline 9 & 3 & 1 & 0.0668 & 3.9 & -0.461 & 1.390 & -0.3231 & -0.2317 & -0.3178 \\
\hline 10 & 3 & 2 & 0.0144 & 3 & -0.838 & -1.390 & -0.5883 & -0.4525 & -0.5805 \\
\hline 11 & 3 & 3 & 0.0563 & 3.7 & -0.536 & 0.772 & -0.3763 & -0.2764 & -0.3705 \\
\hline 12 & 3 & 4 & 0.06 & 3.5 & -0.510 & 0.154 & -0.3577 & -0.2673 & -0.3525 \\
\hline 13 & 4 & 1 & 0.0262 & 3.4 & -0.753 & -0.154 & -0.5284 & -0.3979 & -0.5209 \\
\hline 14 & 4 & 2 & 0.097 & 3 & -0.244 & -1.390 & -0.1712 & -0.1394 & -0.1695 \\
\hline 15 & 4 & 3 & 0.0198 & 3.6 & -0.799 & 0.463 & -0.5607 & -0.4173 & -0.5524 \\
\hline 16 & 4 & 4 & 0.0444 & 3.6 & -0.622 & 0.463 & -0.4364 & -0.3240 & -0.4299 \\
\hline 17 & 5 & 1 & 0.1234 & 3.6 & -0.054 & 0.463 & -0.0375 & -0.0245 & -0.0368 \\
\hline 18 & 5 & 2 & 0.0646 & 2.8 & -0.477 & -2.008 & -0.3349 & -0.2672 & -0.3311 \\
\hline 19 & 5 & 3 & 0.1074 & 3.6 & -0.169 & 0.463 & -0.1183 & -0.0852 & -0.1164 \\
\hline 20 & 5 & 4 & 0.1316 & 3.8 & 0.005 & 1.081 & 0.0040 & 0.0115 & 0.0045 \\
\hline 21 & 6 & 1 & 0.1644 & 3.8 & 0.241 & 1.081 & 0.1696 & 0.1358 & 0.1677 \\
\hline 22 & 6 & 2 & 0.1638 & 3 & 0.237 & -1.390 & 0.1661 & 0.1138 & 0.1630 \\
\hline 23 & 6 & 3 & 0.2304 & 3.6 & 0.716 & 0.463 & 0.5027 & 0.3810 & 0.4957 \\
\hline 24 & 6 & 4 & 0.1198 & 3.6 & -0.080 & 0.463 & -0.0557 & -0.0382 & -0.0547 \\
\hline 25 & 7 & 1 & 0.367 & 3.8 & 1.699 & 1.081 & 1.1926 & 0.9038 & 1.1759 \\
\hline 26 & 7 & 2 & 0.4234 & 2.8 & 2.104 & -2.008 & 1.4767 & 1.0929 & 1.4545 \\
\hline 27 & 7 & 3 & 0.506 & 3.6 & 2.698 & 0.463 & 1.8943 & 1.4257 & 1.8673 \\
\hline 28 & 7 & 4 & 0.4194 & 3.4 & 2.075 & -0.154 & 1.4569 & 1.0925 & 1.4359 \\
\hline
\end{tabular}

Table 3. Principal Component Analysis.

\begin{tabular}{|c|c|c|c|c|c|}
\hline \multirow{2}{*}{ Principal Component } & \multicolumn{3}{|c|}{ Original eigenvalues } & \multicolumn{2}{c|}{ Eigenvectors } \\
\cline { 2 - 6 } & $\begin{array}{c}\text { Eigenvalu } \\
\text { es }\end{array}$ & $\begin{array}{c}\text { Variance } \\
\text { ratio }\end{array}$ & $\begin{array}{c}\text { Cumulative } \\
\text { ratio }\end{array}$ & $Y_{1}$ & $Y_{2}$ \\
\hline 1 & 1.0432 & 0.522 & 0.522 & 0.707 & 0.707 \\
\hline 2 & 0.9568 & 0.478 & 1.000 & 0.707 & -0.707 \\
\hline
\end{tabular}

\subsection{The parameter optimization based on first-order regression prediction model}

According to the experimental data in Table 1, using the MINITAB to establish the first-order regression model of influence factors and response variables, and calculating its predictive ability index by formula (7)

$$
r_{j}=\frac{R_{j}^{2}}{\sum_{i=1}^{p} R_{i}^{2}}
$$

the result is as follows

$Y_{1}=-0.0406+0.0494 x_{1}-0.0104 x_{2}$

$R^{2}=53.1 \%, r_{1}=0.993$

$Y_{2}=3.42-0.0027 x_{1}+0.0171 x_{2}$

$R^{2}=0.4 \%, r_{2}=0.007$

Calculated by formula (6)

$Z_{1}=0.707 \times 0.993 \times Y_{1}+0.707 \times 0.007 \times Y_{2}$

$Z_{2}=0.707 \times 0.993 \times Y_{1}-0.707 \times 0.007 \times Y_{2}$

Calculated by formula (3)

$M P I_{1}=0.522 Z_{1}+0.478 Z_{2}=0.702 Y_{1}+0.0002 Y_{2}$

The main effect chart as shown in Figure 2.

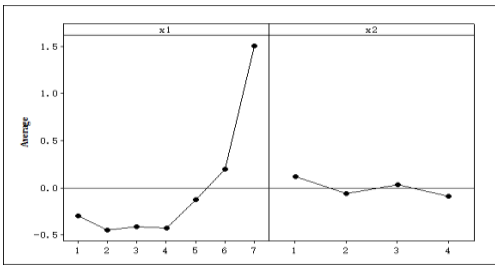

Fig. 2. Main effect chart. 
Since the quality characteristic of $\mathrm{y}_{1}$ and $\mathrm{y}_{2}$ are all smaller for better, so the lower the value of MPI, the better effect of the response. According to figure 2, the minimum value of $\mathrm{x}_{1}$ is second level, and $\mathrm{x}_{2}$ is fourth level. So the result of parameter optimization design by first-order regression prediction model is $(2,4)$.

\subsection{The parameter optimization based on second-order regression prediction model}

According to the experimental data in Table 1, using the MINITAB to establish the second-order regression model of influence factors and response variables, and calculating its predictive ability index by formula (7), the result is as follows

$$
\begin{aligned}
& y_{1}=0.35-0.155 x_{1}-0.0695 x_{2}+0.0113 x_{1} x_{2}+0.022 x_{1} x_{2} \\
& +0.0028 x_{2} x_{2} \\
& R^{2}=87.7 \%, r_{1}=0.746 \\
& +0.171 x_{2} x_{2} \\
& R^{2}=29.8 \%, r_{2}=0.254
\end{aligned}
$$

Calculated by formula (6)

Calculated by formula (3)

$$
\begin{aligned}
& Z_{1}=0.707 \times 0.746 \times Y_{1}+0.707 \times 0.254 \times Y_{2} \\
& Z_{2}=0.707 \times 0.746 \times Y_{1}-0.707 \times 0.254 \times Y_{2}
\end{aligned}
$$

The main effect chart as shown in Figure 3.

$$
M P I_{2}=0.522 Z_{1}+0.478 Z_{2}=0.527 Y_{1}+0.008 Y_{2}
$$

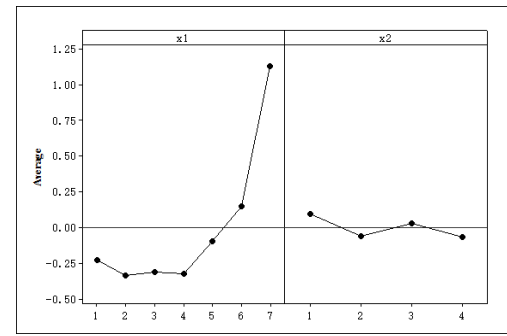

Fig. 3. Main effect chart.

So the result of parameter optimization design by second-order regression prediction model is $(2,4)$.

\subsection{The parameter optimization Based on RBF neural network prediction model}

Above that, the fitting degrees(R2) of the first-order and second-order regression formulas of polymerization process are respectively $53.1 \%, 0.4 \%$ and $87.7 \%, 29.8 \%$, If the R2 less than $60 \%$, it means that the prediction effect of first or second order parameter regression model is not ideal. So the RBF neural network is adopted to establish the nonlinear predictive model and optimize parameter.

Using the MATLAB to establish the RBF neural network model of influence factors and response variables. Two RBF neural network models are established with $x_{1}$ and $x_{2}$ as input variables and $y_{1}$ and $y_{2}$ as output variables respectively. When setting the goal of RBF neural network is 0 and spread is 8 , the MSE of the RBF neural network model is smallest. The MSE of network training are respectively $1.3835 * 10-5$ and $6.3297 * 10-4$. It means that nonlinear prediction model based on RBF neural network is ideal. Calculating its predictive ability index by formula (4), the result is as follows

$\mathrm{r}_{1}=0.9786, \mathrm{r}_{2}=0.0214$

Calculated by formula(6)

Calculated by formula(3)

$$
\begin{aligned}
& Z_{1}=0.707 \times 0.9786 \times Y_{1}+0.707 \times 0.0214 \times Y_{2} \\
& Z_{2}=0.707 \times 0.9786 \times Y_{1}-0.707 \times 0.0214 \times Y_{2}
\end{aligned}
$$

The main effect chart as shown in Figure 4. 


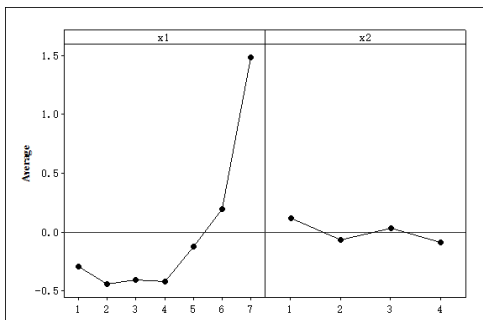

Fig. 4. Main effect chart.

The results of optimization parameters design based on RBF neural network prediction model is also $(2,4)$.

The results of optimization parameters design above the three model are all $(2,4)$. According to the experimental data in Table 1 and compare with the original process parameter $(4,3)$, the result of $(2,4)$ can be seen that the capacitance value and the loss tangent are all improved, and the effect of optimization parameters is achieve to ideal.

\section{Conclusion}

This paper proposed an improved weighted principal component analysis (PCA) method based on parametric and nonparametric prediction models. It is aim to solve the problem of multiple response process parameter optimization design, and this method is applied to optimize the multiple response process parameters in the thermal polymerization process. The results show that this method is effectively improving the two response quality characteristic.

(1)The method based on parametric and non-parametric prediction models to improved weighted principal component analysis, considers the predictive ability of multiple response models. It defined the evaluate the predictive ability index of each response model, and introduces it into the MPI model of the weighted principal component analysis method, which can improve the effectiveness of parameter optimization.

(2)This paper proposed an improved weighted principal component analysis (PCA) method based on RBF neural network prediction model. Because of the RBF neural network has a good generalization ability in nonlinear model. Therefore, in the complex nonlinear production process, it is an effective method to optimization the multiple response process parameter based on RBF neural network prediction model.

\section{Acknowledgments}

The research work was supported by National Natural Science Foundation of China under Grant No. U1404702, Aviation Science Fund under Grant No. 2014ZG55021, Nature Science Foundation of Henan Province under Grant No. 162102210083.

\section{References}

1. Wu F C. Optimization of correlated multiple quality characteristics using desirability function. Quality engineering, 17,1 (2005).

2. J Jun Wang, Y Zhong Ma, Y huan Zhai. Optimal Design of Correlated Multiple quality characteristics. Engineering Management, 25,2 (2011).

3. Ali S, Mahdi B. A new desirability function-based method for correlated multiple response optimization. The International Journal of Advanced Manufacturing Technology, 76 (2015).

4. Liao H C. Multi-response optimization using weighted principal components. The International Journal of Advanced Manufacturing Technology, 27,7 (2006).

5. Shit J S, Tzen Y F, Yang J. B.. Principal components analysis for multiple quality characteristics optimization of mental inert gas welding aluminum foam plate. Materials and Design, 32, 3 (2011).

6. Y Dong Zhang, Z He. Application of Improved Principal Component Analysis Method to the Multi-Response Optimizatio. Modular Machine Tool \& Automatic Manufacturing Technique, 11 (2012).

7. Q An Cui. Design of experiments and global modeling approach based on sub-domains significance for complicated relatonship process. Systems Engineering-Theory\&Practice, 33, 9 (2013).

8. Ali N, Celik T M. Neural network based method for conversion of solar radiation data. Energy Conversion and Management, 67,(2013).

9. X Ming Yan, Z Zheng. Performance comparison between some nonlinear curves fitting with BP and RBF. Journal of Fujian Agriculture and Forestry University (Natural Science Edition), 40,6 (2011).

10. Afantitis A, Melagraki G, Makridima K. et al. Prediction of high weight polymers glass transition temperature using RBF neural networks. Journal of Molecular Structure Theochem, 716, 1(2005). 
11. H Jie Fan. Design and optimization of capacitance decay program for metallized film capacitors. ELECTRONIC COMPONENTS AND MATERIALS, 30,9 (2011). 